\title{
Preparation of Carbon-Copper-Silicon Nanocomposite Materials and Coatings Owing to Abrasive-Reactive Wear
}

\author{
F.Kh. Urakaev ${ }^{a, *}$, T.A. Ketegenov ${ }^{b}$, Y.M. Borzdov ${ }^{a}$, I.V. SAvchenko ${ }^{c}$, \\ V.S. Shevchenko ${ }^{a}$, S.V. StankUs ${ }^{c}$, N.F. UVARov ${ }^{d}$ And N.P. Pokhilenko ${ }^{a}$ \\ ${ }^{a}$ Institute of Geology and Mineralogy SB RAS, Novosibirsk, Russia \\ ${ }^{b}$ Institute of High Technologies NAC "Kazatomprom", Almaty, Kazakhstan \\ ${ }^{c}$ Kutateladze Institute of Thermophysics SB RAS, Novosibirsk, Russia \\ ${ }^{d}$ Institute of Solid State Chemistry and Mechanochemistry SB RAS, Novosibirsk, Russia
}

\begin{abstract}
We will focus on the important aspect of mechanical activation by grinding in a mill, namely, nanoscale wear of the treated substances and of the milling tools. A new technology called abrasive-reactive wear has been developed that utilizes wear debris as an integral component of the reaction system rather than treating it as a harmful impurity. This technology is applied to the processing of low-grade diamond and silicon by cupric milling tools. Abnormal influence of graphite on abrasive wear degree is established.
\end{abstract}

PACS numbers: 81.20.Ev, 81.40.Pq, 81.07.Wx, 81.07.Bc, 81.05.Mh

\section{Introduction}

The mechanical activation (MA) of powders usually carried out in suitably designed ball mills that are considered mechanochemical reactors (MR), essentially consists of the repeated mechanical loading of powders trapped between the colliding surfaces of the milling tools [1]. The local compressive and shear events create hot spots and induce plastic deformation and fracturing of the particles [2-4]. Under such circumstances, powder particles undergo continuous comminution associated with interface renewal processes. These promote the occurrence of physicochemical transformations including the microstructural refinement down to nanometer size $[5,6]$.

Contamination due to the abrasion of milling tools is an inevitable problem of conventional MA in MR; the level of contamination can reach several percent and can rarely be kept below a few tenth of a percent [5]. Many authors mentioned this problem. Moreover, some concrete examples of the possible participation of the milling tools' material in MA processes were reported [7], but these works have not been followed up on.

It is evident that regular research of the participation of a material of milling tools in the course of mechanochemical reactions was not carried out. The prospect of their realization does not cause doubts. As against the traditional realization of mechanochemical reactions in mixes of powders with the sizes of initial particles $\approx 50 \mu \mathrm{m}$, the synthesis of nanocrystalline final

\footnotetext{
* corresponding author; e-mail:

urakaev@uiggm.nsc.ru; urakaev@igm.nsc.ru
}

products proceeds for hundreds of hours of MA [8], and owing to participation of the nanosized material resulting from the abrasive wear of milling tools [7, 9], a significant reduction of this time could be expected. Intensive research of mechanochemical reactions was recently began with the participation of a material of milling tools not only confirmed this assumption, but also revealed many other advantages and prospects of their realization.

Sometimes it is possible to select the material of the milling tools in such a way that the wear debris becomes a beneficial component of the intended product. We plan to carry this possibility to the extreme: We will develop a new technique called abrasive-reactive wear (ARW) that uses the material of the milling tools as a reactant that becomes a major component of the product, not just a tolerable impurity $[9,10]$. This innovative new approach opens the door to applications of the ARW phenomenon for processing different materials. The probable scopes of the ARW method stated in the paper lead us to believe that it can find specific application in various areas of modern science.

$>$ From the point of view of enhancing the possibilities of the ARW method, the use of diamond and silicon is of special interest owing to its unique physicochemical and abrasive properties and ability to interact with transitional metals and alloys, i.e., a combination of properties of both abrasives and reagents [11, 12]. It should also be noted that mechanochemical synthesis could in the future provide a more efficient utilization of both natural diamond and synthetic silicon as the result of introducing the low-grade materials into the technological process.

A specific example of a property of technological relevance that characterizes materials prepared by techniques studied in the present paper is the heat conduc- 
tivity. Linked to this, the problem of heat removal is very important for the semiconductor industry. For this reason, the improvement of thermal properties of materials used for manufacturing microelectronics components becomes crucial [13-16]. The USA "ADS" company offered nanocomposite materials on a $\mathrm{Cu}$ and diamond basis that have higher heat conductivity in comparison with traditionally used materials on a $\mathrm{Cu}$ or $\mathrm{Al}$ basis [17]. Moreover, particles of diamond in copper-diamond composites show a high degree of stability to graphitization under sintering at temperatures up to $1150-1250 \mathrm{~K}$ and thus this composite material can be used for the creation of contacts and high-current electric contacts in the low-voltage equipment [18].

In the present paper nanoscale ARW of the diamond-graphite-silicon system and copper milling tools of MR is applied to the preparation of composites and coatings.

\section{Experimental details}

We used 2-drum AGO-2, EI-2 × 150 and pulverisette-6 mono-drum planetary mills to carry out the MA [9-12]. In order to realize the task formulated above, we constructed rod duralumin drums adapted for utilization in all mills. The drums were equipped with cylindrical inserts made up of copper rod and plate. The movable milling tools were prepared from copper rod and $3 \mathrm{~mm}-$ -thick sheet. The drums can also be adapted to the preparation of coatings: copper plate $(1 \mathrm{~mm}$-thick $)$ is attached to the wall of the inserts; the impacts by the copper milling tools activate the surface of the plate, deliver particles from the powder charge and pound them onto or into the surface.

The natural diamond (purity $>99 \% ; 1.5 \div 0.5 \mu \mathrm{m}$; $1.5 \div 10 \mathrm{~g}$ ) was used to prepare the copper-diamond composites and coatings, while the rhombohedral (R-3 m, see Powder Diffraction File PDF 75-2078) graphite powder (Alfa Aesar, $99.9995 \% ; 1.5 \div 10 \mathrm{~g}$ ) and a mixture of diamond and graphite powders $(3 \div 20 \mathrm{~g})$ were used to prepare the copper-graphite and copper-diamond-graphite composites and coatings. The silicon powder was made from single crystal by the ARW technique (preliminary grinding of the crystals with using copper milling tools). The silicon powder was used to prepare the copper-silicon, copper-silicon-diamond, copper-silicon-graphite, and copper-silicon-diamond-graphite composites and coatings. The equipment for synthesis of diamonds [19] is modified to obtain sintered pellets with diameter of $\approx 6 \mathrm{~mm}$ and thickness up to $3 \mathrm{~mm}$ for electrical and heat conductivity measurements.

MA was performed in an air proof condition up to $120 \mathrm{~min}$. The surfaces of the milling tools turned out to be coated with the MA powder. Compositions and structures of samples were studied by gravimetric, Raman spectroscopy (Dilor/OMARS), X-ray phase analysis (DRON-3), differential thermal analysis (Q-1000), optic (NU-2E), and scanning microscopy (JSM-6380 and LEO-1550).

\section{Results and discussion}

The results of the experiment demonstrated both similarities and dissimilarities of MA only for diamond (sample 1, S1), graphite (S2), silicon (S3), and the diamond-silicon (S4), diamond-graphite (S5), silicon-graphite (S6), diamond-silicon-graphite (S7) mixtures. In all the cases, the movable copper milling tools were subjected to rolling and transformed into ellipsoids. However, contrary to expectations, the wear of copper milling tools after the MA of samples 1-4 appeared nearly one order of magnitude lower than that of samples 5-7 (abnormal influence of graphite containing systems on abrasive wear degree is established, cf. images in Fig. 10 at the end). Examination of working surfaces of milling tools with optical microscopy showed that the change in mass of the copper inserts is related to the phenomenon of hardening, i.e., uniform penetration of solid diamond particles (S1) or the diamond-graphite mixture (S5) into the working layer of inserts with coatings formation. This phenomenon hampered the determination of the exact composition of the newly formed composites by the weight analysis. For example [11], based on the masses of milling tools weighed before and after the MA of S1, the composite corresponds to a diamond content of $75.8 \%$ (the $\mathrm{Cu}$ content is $24.2 \%$ ). After the MA of S5, the content of carbon (diamond and graphite) in the composite was $22.6 \%$ and the $\mathrm{Cu}$ content was $77.4 \%$. We could solve this problem by X-ray phase analysis (XRD, Fig. 1-5), Raman spectroscopy (Fig. 6), and differential thermal analysis (Fig. 7).

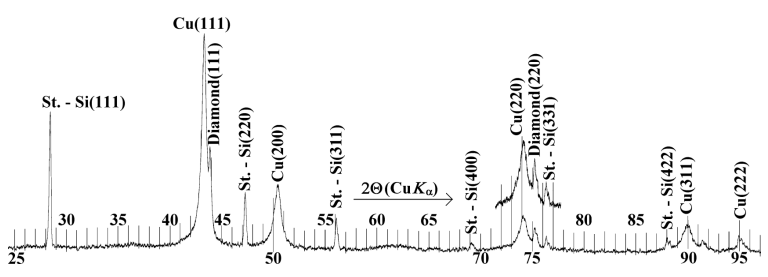

Fig. 1. X-ray phase analysis of the product of MA of diamond (4.192 g, pulverisette-6, P6) for $120 \mathrm{~min}$, sample 1 (S1). St. - lines of standard crystals (powder of carbonyl silicon). $2 \Theta(\mathrm{Cu} K \alpha): \mathrm{Cu} K \alpha$ is the radiation of powder diffractometer DRON-4; $2 \Theta$ is the scattering angle (degree) at the rate of counter movement $0.25 \mathrm{deg} / \mathrm{min}$.

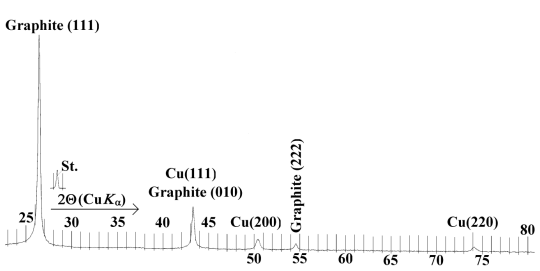

Fig. 2. XRD of MA of graphite $(5.090$ g, P6, 120 min S2). 


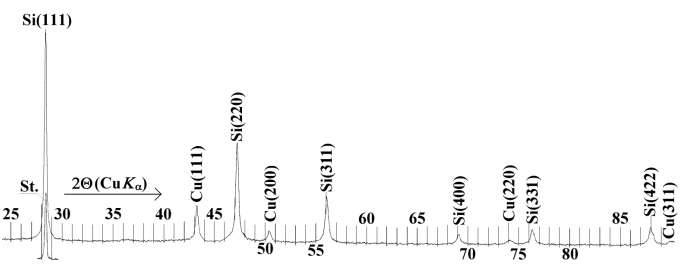

Fig. 3. XRD of MA of silicon $(3.100$ g, P6, $120 \mathrm{~min} \mathrm{S3).}$

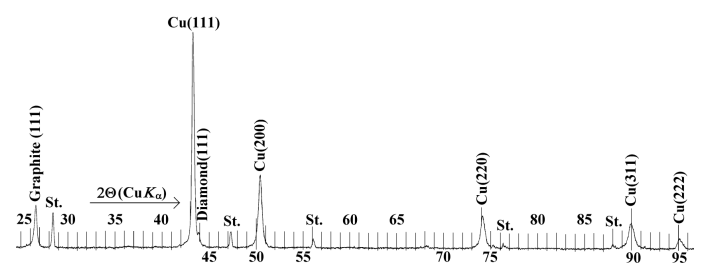

Fig. 4. XRD of MA of diamond (10.244 g) - graphite (10.128 g) mixture (120 min P6, S5).

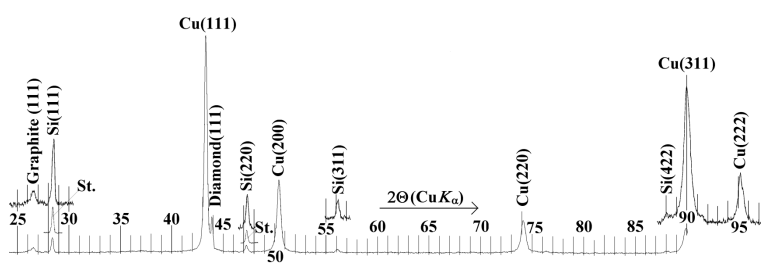

Fig. 5. XRD of MA of diamond (4.197 g) - graphite (5.418 g) silicon (5.715 g) mixture (120 min P6, S7).

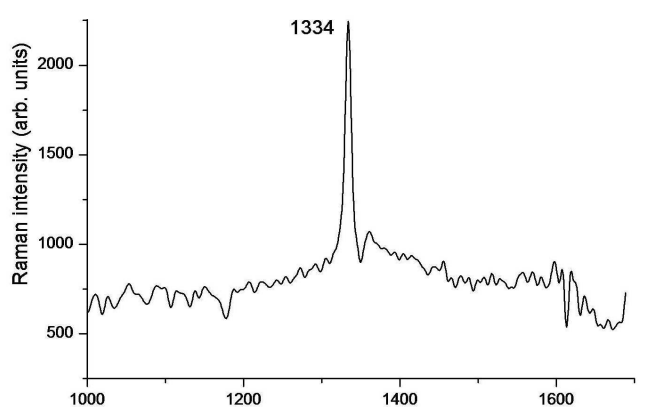

Fig. 6. Raman spectrum of the MA diamond (mill EI- $\times 150,210 \mathrm{~min}, \mathrm{~S} 1$, see [12]). The diamond line on the $x$-axis is $1334 \mathrm{~cm}^{-1}$.

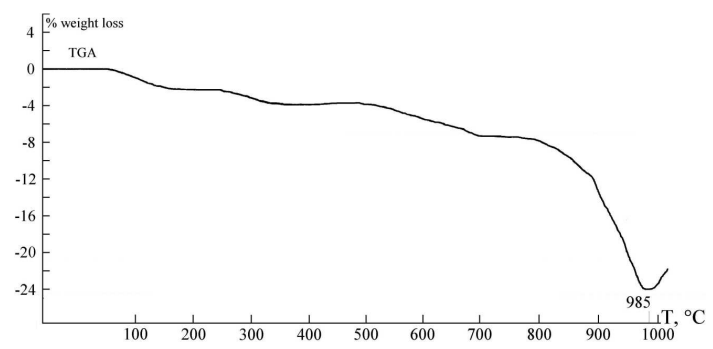

Fig. 7. Thermogravimetry (TGA) of MA of diamond $(1.5 \mathrm{~g})$ - graphite $(1.5 \mathrm{~g})$ mixture $(210 \mathrm{~min}$, sample 5$)$ in the atmosphere [12].

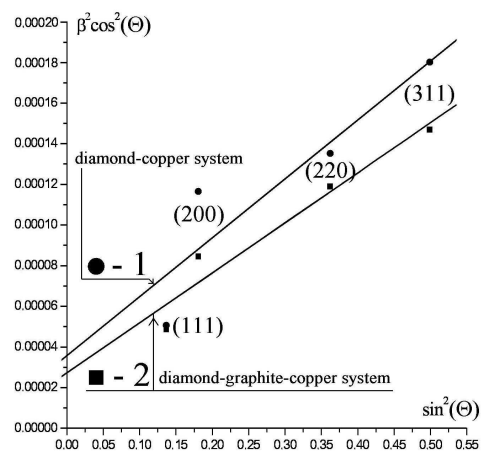

Fig. 8. The processing of XRD data on $\mathrm{Cu}$ lines by the Williamson-Hall method [12]: 1 - sample 1 (S1); 2 - sample 5 (S5).

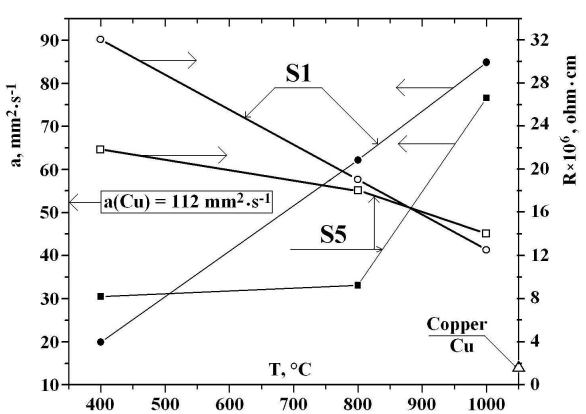

Fig. 9. Thermal conductivity (a in $\mathrm{mm}^{2} \mathrm{~s}^{-1}$ ) and specific resistance $(R$ in $\Omega \mathrm{cm})$ sintering (pellets) obtained from the MA samples $\mathrm{S} 1$ and $\mathrm{S} 5$ in [12]at the following temperatures $T$ : $400{ }^{\circ} \mathrm{C} ; 800{ }^{\circ} \mathrm{C}$; and $1000^{\circ} \mathrm{C}$. Arrows indicate the relevant axis measuring the properties of pellets.

The powder XRD data on diamond, graphite, silicon and their mixtures were identical with PDF 79-1467 for diamond, PDF 75-2078 for R-3m graphite, PDF 27-1402 for silicon and the superposition of all patterns for the mixtures. After the MA of samples, the XRD data virtually did not differ under identical measurement conditions (Fig. 1-5): diamond crystals remain in the crystalline state, but the reflections are strongly masked by reflections of nanowear particles of the copper furniture

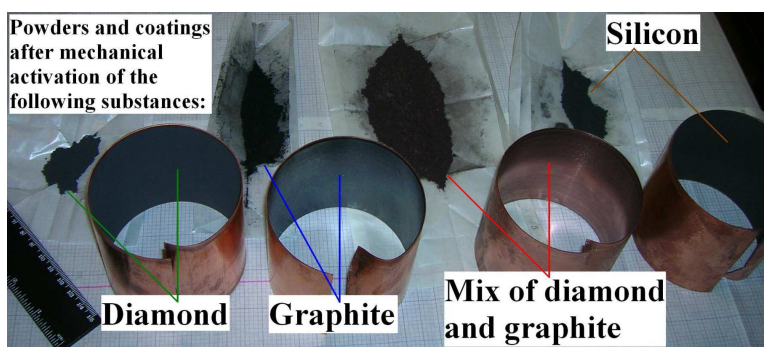

Fig. 10. MA results of the samples (S1-S3, S5) and obtained coatings and composites. 
of milling tools; hence, the MA of all samples does not produce carbon (or silicon) and copper compounds (it is well known that copper does not react with carbon and silicon) and the newly formed composites have a copper-carbon-silicon composition (the particles of the new formed materials are constituted of nanodomains of $\mathrm{C}, \mathrm{Cu}$ and $\mathrm{Si}$ ). Raman spectrometric data on the starting diamond (or silicon) and its MA product (Fig. 6) indicate that MA does not create chemical bonds between copper and carbon (or silicon) atoms $(\mathrm{Cu}-\mathrm{C}$ or $\mathrm{Cu}-\mathrm{Si}$ bonds). Analysis of samples, as well as the control sample of powder mixture of diamond, graphite, silicon and copper, in atmosphere by the thermogravimetry (TGA, heating rate $10^{\circ} \mathrm{C} / \mathrm{min}$ ) yielded important information (Fig. 7). The results show that a significant variation in the sample mass begins at $T>700{ }^{\circ} \mathrm{C}$ (burning of diamond) for $\mathrm{S} 1$, at $T>100^{\circ} \mathrm{C}$ (burning of MA graphite) for $\mathrm{S} 2$, and at $T>400^{\circ} \mathrm{C}$ (oxidation of silicon) for $\mathrm{S} 3$. Based on a TGA pattern similar to that in Fig. 7, the mass of the control mixture also increases markedly due to oxidation at $T \approx 1000{ }^{\circ} \mathrm{C}$ (the presence of carbon creates a reducing environment in the crucible and hampers the oxidation of copper). Therefore, the TGA data can be used to decipher constituents of composites. For example, based on the TGA data (Fig. 7), the composite in S5 includes $24 \%$ carbon $(7.3 \%$ graphite and $16.7 \%$ diamond). Hence, the $\mathrm{Cu}$ content should be $76 \%$, which is very close to the weight analysis data. For comparison, the TGA data indicate that the MA product in S1 contains $77 \%$ diamond and $23 \% \mathrm{Cu}$.

Data on the microanalysis of different points of samples confirmed qualitatively the XRD data. In terms of quantitative assessment, the images obtained (here not reported) indicate the presence of some heterogeneity in the distribution of carbon and silicon. Light-colored areas correspond to C-rich zones, while dark areas indicate C-poor zones. The images also show areas with an intermediate composition. SEM images of the samples show that the copper-carbon composites mainly include agglomerates $5-10 \mu \mathrm{m}$ in size. Higher resolution images show that the agglomerates can be composed of particles much smaller than $1 \mu \mathrm{m}$. In order to determine the characteristics of the state of copper in the MA powder samples, we processed the XRD data using the WilliamsonHall plot $[5-12,20]: \beta^{2} \cos ^{2} \Theta=(\Lambda / D)^{2}+16 \varepsilon^{2} \sin ^{2} \Theta$, where $\beta$ is the broadening of reflections after the subtraction of the individual broadening of reflections of the standard (carbonyl silicon), $(\Lambda / D)^{2}$ is the Y-intercept, $\Lambda=0.15418 \mathrm{~nm}$ is the wavelength of the $\mathrm{Cu} K_{\alpha}$ radiation, and $D$ is the dimension of crystallites. Tangent $\operatorname{tg} A=16 \varepsilon^{2}$ of slope A of straight lines 1 and 2 yields the micro strain $\varepsilon$ value of crystallites. Processing of data in Fig. 15 and materials pertaining to the analogous (in terms of MA) S1 and S5, see Fig. 8, yielded the following results:

S1 (copper-diamond system): $D_{\mathrm{Cu}}=246 \AA \approx 25 \mathrm{~nm}$, $\varepsilon_{\mathrm{Cu}}=0.0021(0.21 \%)$; the composite in S1 includes $63.3 \% \mathrm{Cu}$ (the missing mass $2.8 \mathrm{~g}$ of diamond was spent on a covering of a surface of milling tools);

S2 (copper-graphite system): $D_{\mathrm{Gr}}=380 \AA \approx 38 \mathrm{~nm}$, $\varepsilon_{\mathrm{Gr}}=0.0008(0.08 \%) D_{\mathrm{Cu}}(111) \approx 20 \mathrm{~nm}$; the composite in $\mathrm{S} 2$ includes $32.5 \% \mathrm{Cu}$ (the missing mass $0.9 \mathrm{~g}$ of graphite was spent on a covering of the surface);

S3 (copper-silicon system): $D_{\mathrm{Si}}=462 \AA \approx 46 \mathrm{~nm}$, $\varepsilon_{\mathrm{Si}}=0.0020(0.2 \%) D_{\mathrm{Cu}}(111) \approx 34 \mathrm{~nm}$; the composite in S3 includes $47.5 \% \mathrm{Cu}$ (the missing mass $1.7 \mathrm{~g}$ of silicon was spent on a covering of the surface);

S5 (copper-diamond-graphite system): $D_{\mathrm{Cu}}=243 \AA$ $\approx 24 \mathrm{~nm} \varepsilon_{\mathrm{Cu}}=0.0011(0.11 \%) D_{\mathrm{Gr}}(111) \approx 34 \mathrm{~nm}$; the composite in $\mathrm{S} 5$ includes $80.5 \% \mathrm{Cu}$ (the missing mass $1.2 \mathrm{~g}$ of the MA diamond-graphite mixture was spent on a covering of the surface);

S7 (copper-diamond-graphite-silicon system): $D_{\mathrm{Cu}}=$ $440 \AA \approx 44 \mathrm{~nm} \varepsilon_{\mathrm{Cu}}=0.0025(0.25 \%) D_{\mathrm{Gr}}(111) \approx 24 \mathrm{~nm}$; $D_{\mathrm{Si}}(111) \approx 56 \mathrm{~nm} D_{\mathrm{Si}}(220) \approx 52 \mathrm{~nm}$; the composite in $\mathrm{S} 7$ includes $84.7 \% \mathrm{Cu}$ (the missing mass $0.9 \mathrm{~g}$ of the MA diamond-graphite-silicon mixture was spent on a covering of the surface).

Based on the high-resolution SEM data, the relatively large agglomerates of particles have a cauliflower-type internal structure with block dimension similar to certain dimensions of crystallites. The results for the MA graphite containing mixtures suggest that graphite can be used to produce a amorphous [12] and nonautonomous [21] phase on the surface of other particles of the composite (non-autonomous phases are surface states which cannot exist as individual phases separately from the support; typical examples of non-autonomous phases are Langmuir-Blodgett adsorption layers or surface oxides which are characterized by intrinsic quasi two-dimensional phase diagrams or other physical and chemical properties strongly different from those of bulk pure compounds). Study of several physicochemical properties of composites demonstrated that the nonautonomous phase has a significant influence on the process of MA. In particular, the formation of amorphous carbon enhances the degree of nanowear of the copper material of milling tools.

In Fig. 9 data on measurement (at room temperature) thermal conductivity (a) and specific resistance $(R)$ for sintered (under the pressure of $\approx 3.5 \mathrm{GPa}$ ) products (pellets) are shown depending on the sintering temperature of S1 and S5. It is visible, that there is a concurrent change in the data $(a)$ and $(1 / R)$ for both samples. Now as much as possible reached by us thermal conductivity of the copper-diamond composite has made $\approx 140 \mathrm{~mm}^{2} \mathrm{~s}^{-1}$.

ARW can also be adapted to the preparation of coatings [22]. For example, if copper plate is attached to the wall of the milling container, the impacts by the copper milling tools activate the surface of the plate, deliver particles from the powder charge and pound them onto or into the surface. The structure and properties of the coating depend on the MA conditions. Some experimental aspects of the preparation of coatings (deposition of elements or their mixtures on a surface of the copper $1 \mathrm{~mm}$-thick sheets) are shown in Fig. 10. 


\section{Conclusion}

We have substantiated a new field of low-grade materials (diamond, silicon, etc.) utilization, namely an abrasive-reactive synthesis based on the copper material of the milling tools of a planetary mill. A scrap of any metal and ceramic products can be used as milling tools, which makes it possible to considerably extend the potential of the abrasive-reactive wear method and the range of treated compounds.

\section{Acknowledgments}

The support through the Scientific Research Works within the framework of the Federal Program The scientific and pedagogical staff of the innovative Russia/State contract No. 02.740.11.0109 is gratefully acknowledged.

\section{References}

[1] F.Kh. Urakaev, V.V. Boldyrev, Powder Techn. 107, 93 (2000).

[2] F.Kh. Urakaev, V.V. Boldyrev, Powder Techn. 107, 197 (2000).

[3] F.Kh. Urakaev, Phys. Chem. Minerals 34, 351 (2007).

[4] F.Kh. Urakaev, Phys. Chem. Minerals 35, 231 (2008).

[5] C. Suryanarayana, Mechanical Alloying and Milling, Marcel Dekker, New York 2004.

[6] F.Kh. Urakaev, V.S. Shevchenko, Russian J. Phys. Chem. 80, 157 (2006).

[7] F.Kh. Urakaev, Int. J. Mineral Proc. 92, 58 (2009).

[8] J.Z. Jiang, R.K. Larsen, R. Lin, S. Mørup, I. Chorkendorff, K. Nielsen, K. Hansen, K. West, J. Solid State Chem. 138, 114 (1998).
[9] F.Kh. Urakaev, V.S. Shevchenko, KONA 25, 162 (2007).

[10] F.Kh. Urakaev, T.A. Ketegenov, E.I. Petrushin, Yu.P. Savintsev, O.A. Tyumentseva, A.P. Chupakhin, V.S. Shevchenko, T.S. Yusupov, V.V. Boldyrev, J. Mining Sci. 39, 304 (2003).

[11] F.Kh. Urakaev, Yu.N. Pal'yanov, V.S. Shevchenko, N.V. Sobolev, Doklady Earth Sci. 395, 214 (2004).

[12] F.Kh. Urakaev, V.S. Shevchenko, A.M. Logvinova, I.A. Madyukov, E.I. Petrushin, T.S. Yusupov, N.V. Sobolev, Doklady Earth Sci. 415, 755 (2007).

[13] K. Yoshida, H. Morigami, Microelectronics Reliability 44, 303 (2004).

[14] T. Tyler, O. Shenderova, G. Cunningham, J. Walsh, J. Drobnik, G. McGuire, Diamond and Related Materials 15, 2078 (2006).

[15] S.V. Kidalov, F.M. Shakhov, A.Ya. Vul', Diamond and Related Materials 16, 2063 (2007).

[16] K. Raleva, D. Vasileska, S.M. Goodnick, IEEE Electron Device Letters 29, 621 (2008).

[17] http://www.advanceddiamond.com/products.html .

[18] W.Z. Shao, V.V. Ivanov, L. Zhen, Y.S. Cui, Y. Wang, Materials Letters 58, 146 (2003).

[19] A.G. Sokol, Yu.N. Pal'yanov, Contributions to Mineralogy and Petrology 155, 33 (2008).

[20] O. Boytsov, F. Bernard, E. Gaffet, Y. Champion, A. Ustinov, Chemistry for Sustainable Development 13, 131 (2005).

[21] N.F. Uvarov, Russian Chemical Reviews 76, 415 (2007).

[22] Á. Révész, L. Takacs, Surface and Coatings Technology. 203, 3026 (2009). 